\title{
Chapter 2 \\ Model predictive control for combined water supply and navigability/sustainability in river systems
}

\author{
V. Puig, C. Ocampo-Martinez and R.R. Negenborn
}

\begin{abstract}
In this chapter, a methodology for the optimal management of combined water supply and navigability/sustainability in river systems based on model predictive control (MPC) is proposed. A control-oriented modeling methodology for this type of systems is presented as well. MPC is used to generate flow-control strategies from the sources to both the farmers and urban consumers in order to meet future demands with appropriate flows, optimizing operational goals such as network safety volumes in dams and smooth operations of actuators (valves, gates and pumps). At the same time, the generated flow-control strategies should allow maintaining the appropriate river water levels that, in turn, allows to preserve the ecological flows and the navigability of the downstream part of the river. The case study of the Guadiana river is used to show and verify the proposed optimal management methodology.
\end{abstract}

\footnotetext{
V. Puig

Institut de Robòtica i Informàtica Industrial (CSIC-UPC), Universitat Politècnica de Catalunya, Barcelona, Spain, e-mail: vpuig@iri.upc.edu

C. Ocampo-Martinez

Institut de Robòtica i Informàtica Industrial (CSIC-UPC), Universitat Politècnica de Catalunya, Barcelona, Spain, e-mail: cocampo@iri.upc.edu

R.R. Negenborn

Delft University of Technology, Delft, The Netherlands e-mail: r.r.Negenborn@tudelft.nl
} 


\subsection{Introduction}

Water management is a subject of increasing concern. Limited water supplies, conservation and sustainability policies, as well as the infrastructure complexity for meeting consumer demands with quality levels make water management a challenging problem. Water systems, which are quite often of large-scale nature, comprise:

- Supplies, where raw water is drawn from superficial or underground sources, such as rivers, reservoirs or boreholes;

- Production facilities, where water is treated to meet consumer-use standards;

- Transport systems, natural or artificial open-flow canals carrying water from the sources to the treatment locations and to the distribution areas;

- Distribution areas, including consumer demands, storage tanks and pressurized pipe networks, to which water must be supplied with appropriate pressure levels

- Control elements in all the above-mentioned subsystems, such as gates, valves, and pumps.

Water supply, treatment, transport and distribution are often operated separately by different authorities and/or utilities. Planning and management of these subsystems have different goals and time scales. Additionally, hydraulics involved differ considerably from one to another, in particular, between large and spatiallydistributed open canals and pressurized water sections for distribution to consumers. In many water systems, network operation is carried out based on heuristic approaches, operator judgment, among other approaches, which may be quite complex for large-scale interconnected systems. Decision support systems, which are based on mathematical network and operation models, may efficiently contribute to the optimal management of water networks by computing control strategies ahead in time, which optimize management goals [3]. Optimization and optimal control techniques provide an important contribution to strategy computation in water systems management, as reported in [1, 14].

Many modern water systems are operated through centralized or distributed telemetry and telecontrol systems but supervised by expert humans. Over the past few years, Model Predictive Control (MPC) has proven to be one of the most effective and accepted control strategies for large-scale complex systems [11, 18]. The objective of using this technique for controlling water systems is to compute, in a predictive way, the manipulated inputs in order to achieve the optimal performance of the network according to a given set of control objectives and predefined performance indices. As shown in [9, 16], MPC controllers are suitable to be used in the global/supervisory control of networks related to the urban water cycle. Figure 2.1 shows a conceptual scheme for a hierarchical structure considered on the control of such networks. Here, the MPC, as the global control law, determines the references (set-points) for the local controllers placed at different elements of the networked system. These references are computed according to measurements taken from sensors distributed around the network. The management level provides the MPC with its operational objectives, which are reflected in the controller design as the performance indices to be enhanced, which can be either minimized or maximized, 


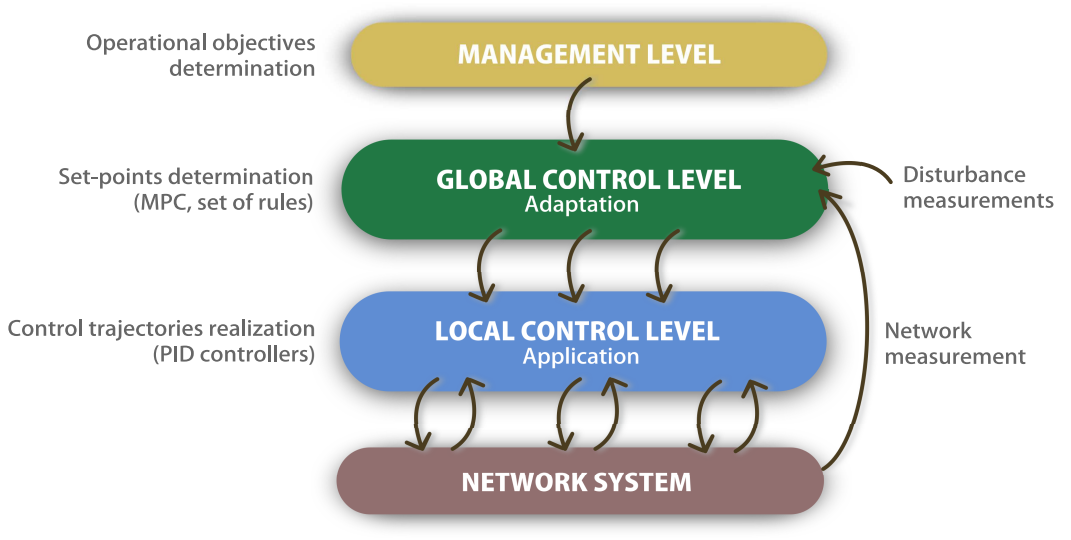

Fig. 2.1: Hierarchical structure for RTC system based on MPC (taken from [14]).

depending on the case. Finally, water systems control requires the use of a supervisory system to monitor the performance of the different control elements in the networks (e.g., valves and gates) and to take appropriate correcting actions in the case where a malfunction is detected, to achieve a proper fault-tolerant control.

In this chapter, a methodology based on MPC for the optimal management of water supply in river systems combined with navigability and sustainability features is proposed. A control-oriented modeling approach for these systems is also presented. MPC is used to generate flow-control strategies from the sources to both the farmers and urban consumers to meet future demands with appropriate flows, optimizing operational goals such as network safety volumes in dams and control input smoothness in actuators. Simultaneously, the generated flow-control strategies should allow maintaining the appropriate water levels in the river, allowing in turn to preserve the ecological flows and the navigability at the downstream portion of the river.

Regarding the case study through which the proposed methodology is illustrated, a brief description is going to be presented next. The Guadiana River is an international river defining a long stretch of the Portuguese-Spanish border, separating Extremadura and Andalucia (Spain) from Alentejo and Algarve (Portugal), see Figure 2.2. The basin of the river extends from the eastern portion of Extremadura to the southern provinces of the Algarve; the river and its tributaries flow from east to west, then south through Portugal to the border towns of Vila Real de Santo António (Portugal) and Ayamonte (Spain), where it flows into the Gulf of Cádiz. With a course that covers a distance of $829 \mathrm{~km}$, it is the fourth-longest in the Iberian peninsula, and its hydrological basin extends over an area of approximately $68,000 \mathrm{~km}^{2}$ (the majority of which lies within Spain). 


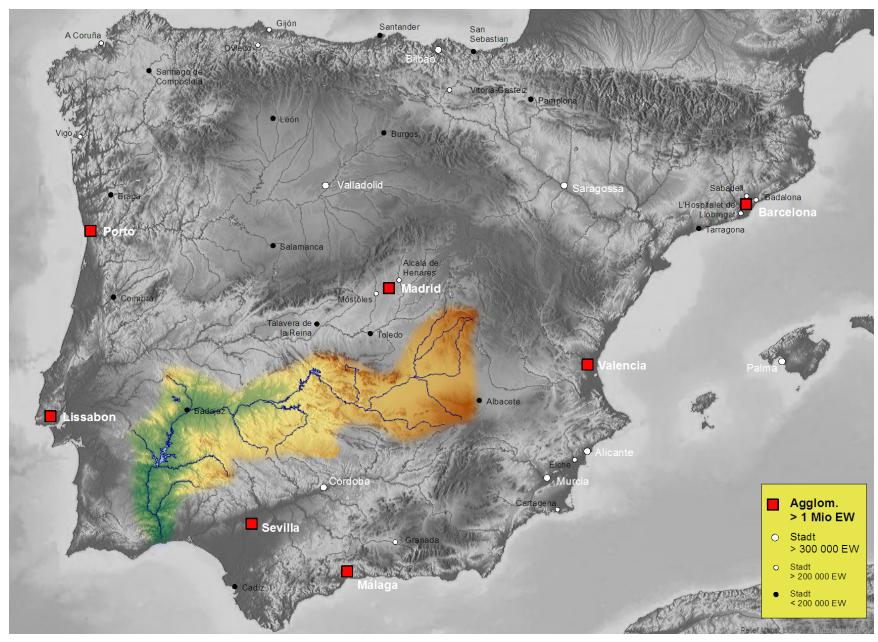

Fig. 2.2: Guadiana Basin.

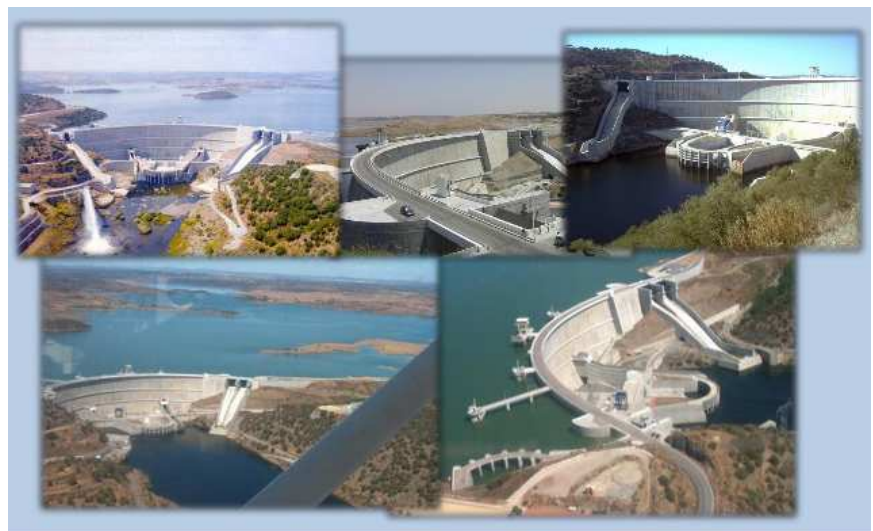

Fig. 2.3: Alqueva Dam.

The Guadiana ecosystem has Mediterranean hydrological characteristics, including high variation in intra- and inter-annual discharge, large floods and severe droughts. This variability is a consequence of considerable variation in rainwater supply, averaged around an annual mean of $400-600 \mathrm{~mm}$. There are over thirty dams on the river basin, the largest of which is the Alqueva Dam, near Moura, in the Beja District, responsible for the largest reservoir in Western Europe (see Figure 2.3. The Alqueva reservoir occupies an area of $250 \mathrm{~km}^{2}$, with a capacity for $4150 \mathrm{hm}^{2}$. For the most part, the Guadiana is navigable until Mértola (a distance of $68 \mathrm{~km}$ ), see Figure 2.4

The chapter is organized as follows. In Section 2.2 the control-oriented modeling methodology is described and the predictive control strategy applied to wa- 


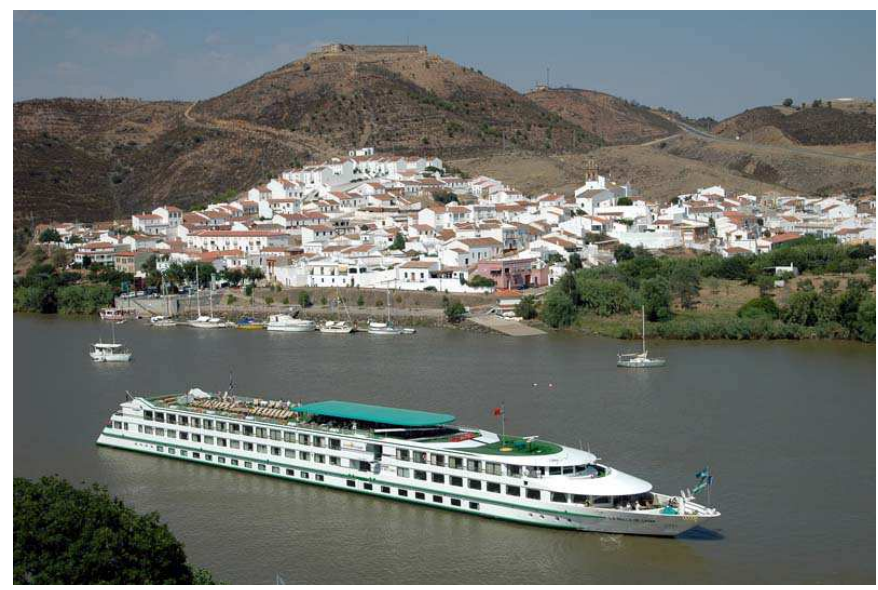

Fig. 2.4: Ship navigation in the Guadiana River.

ter supply systems is presented and discussed for the mentioned case study: the Guadiana river. Section 2.3 highlights and motivates some discussion about the approach proposed in this chapter within the transport of water framework and its relation/connection with the transport over water. Finally, in Section 2.4, the main conclusions are drawn.

\subsection{Proposed approach and case study}

\subsubsection{Control-oriented modeling methodology}

Several modeling techniques dealing with the operational control of water systems have been presented in the literature, see [1, 10], among others. Here, a flow controloriented modeling approach is outlined, which follows the principles presented by the authors in [13, 14]. Natural extensions to include pressure features in the system model can be found in [5, 6, 21, 24]. A water system generally contains storing elements, which store the water coming from the network sources, a network of pressurized/unpressurized pipes and a number of sinks. Valves, gates and/or pumping stations are elements that allow to manipulate the water flow according to a specific policy and to supply water requested by the network users. These flows are chosen by a global management strategy.

The water system model can be considered as composed of a set of constitutive elements, which are presented and discussed below. Some of these elements follow the description reported in [15] for flow networks, but will be included here for the proper completeness of the discussion. 


\section{Tanks and dams}

Water dams/reservoirs provide the entire water system with the storage capacity of water while water tanks at distribution level additionally provide at appropriate elevation levels to supply water with adequate pressure service to consumers. The mass balance expression relating the stored volume $v$, the manipulated inflows $q_{\text {in }}^{j}$ and outflows $q_{\text {out }}^{h}$ (including the demand flows as outflows) for the $i$-th tank can be written as the discrete-time difference equation

$$
v_{i}(k+1)=v_{i}(k)+\Delta t\left(\sum_{j} q_{\mathrm{in}}^{j}(k)-\sum_{h} q_{\mathrm{out}}^{h}(k)\right),
$$

where $\Delta t$ denotes the sampling time and $k$ denotes the discrete-time instant. The physical constraint related to the range of admissible water in the $i$-th dam/tank is expressed as

$$
\underline{v}_{i} \leq v_{i}(k) \leq \bar{v}_{i}, \quad \forall k,
$$

where $\underline{v}_{i}$ and $\bar{v}_{i}$ denote the minimum and the maximum admissible storage capacity, respectively. As this constraint is physical, it is impossible to send more water to a tank than it can store, or drawing more water than the stored amount. Although $\underline{v}_{i}$ might correspond with an empty dam/tank, in practice this value can be set as nonzero in order to maintain an emergency stored volume enough to supply for facing extreme circumstances. Moreover, there will be restrictions in the amount of flow that can be extracted from the dam/tank depending on the volume stored according to the discharge curves.

For simplicity purposes, the dynamic behavior of these elements is described as a function of the volume. However, in most of the cases the measured variable is the water level (by using level sensors), which implies the computation of the water volume taking into account the element geometry.

\section{Actuators}

Several types of control actuators are considered: valves, gates and pumps (more precisely, complex pumping stations). It is assumed that the MPC controller provides the flow set-point to a local controller that is responsible to establish the required flow through the actuator by using a closed-loop control system with a PID or a PLC. The manipulated flows through the actuators represent the manipulated variables, denoted as $q_{u}$. All considered actuators have lower and upper physical limits, which are taken into account as system constraints. As in 2.2., they are expressed as

$$
\underline{q u}_{i} \leq q_{u_{i}}(k) \leq \overline{q_{u}}, \quad \forall k,
$$

where $\underline{q u}_{i}$ and $\overline{q_{u}}$ denote the minimum and the maximum flow capacity, respectively. 


\section{Nodes}

These elements correspond to the network points where water flows are merged or split. Thus, the nodes represent mass balance relations, being modeled as equality constraints related to inflows (from other dams/tanks through gates/valves or pumps) and outflows, these latter being represented not only by manipulated flows but also by demand flows. The expression of the mass conservation in these elements can be written as

$$
\sum_{j} q_{\mathrm{in}}^{j}(k)=\sum_{h} q_{\mathrm{out}}^{h}(k) .
$$

From now on and with some abuse of notation, node inflows and outflows are denoted by $q_{\text {in }}$ and $q_{\text {out }}$, respectively, despite they can be manipulated flows and hence denoted by $q_{u}$, if correspond.

\section{River/canal reaches}

In the proposed control-oriented methodology, a single river/canal reach can be approximated by using the IDZ model introduced in [7] used the Laplace transform

$$
Y_{d n s}(s)=G_{1}(s) Q_{u p s}(s)+G_{2}(s) Q_{d n s}(s),
$$

where $Y_{d n s}(s)$ is the water level at the control point, and $Q_{u p s}(s), Q_{d s n}(s)$ are the upstream and downstream flows, respectively. Moreover, $G_{1}(s)=e^{-\tau_{d} s} / A_{d} s$ and $G_{2}(s)=-1 / A_{d} s$ with $\tau_{d}$ being the downstream transport delay and $A_{d}$ the downstream backwater area.

Taking into account the linearized relation between $Q_{d n s}$ and $Y_{d n s}$ in the control point, the following relation can be established:

$$
Q_{d n s}(s)=\beta Y_{d n s}(s),
$$

where $\beta$ is a constant varying with the operating point. Combining (19.4) and 2.6, the following first order plus time delay (FOPTD) model is obtained

$$
G(s)=\frac{Q_{d n s}(s)}{Q_{\text {ups }}(s)}=\frac{K e^{-\tau_{d} s}}{T s+1},
$$

with $K=1$ and $T=A_{d} / \beta$. This model can be represented in discrete time, using a sampling time $\Delta t$, in terms of the Z-transform as follows:

$$
G_{d}(z)=\frac{Q_{d n s}(z)}{Q_{u p s}(z)}=\frac{b_{0} z^{-d}}{z-a_{1}},
$$

where $d=\tau_{d} / T_{s}, b_{0}=1-a_{1}$ and $a_{1}=e^{-\frac{T_{s}}{T}}$. Alternatively, it can be written as a difference equation as 


$$
q_{d n s}(k+1)=a_{1} q_{d n s}(k)+b_{0} q_{u p s}(k-d) .
$$

\section{Urban and irrigation demands}

Urban and irrigation demands are considered as measured disturbance of the system at a given time instant. The demand in urban areas can be anticipated by a forecasting algorithm that is integrated within the MPC closed-loop architecture [17, 23]. In [17], the demand forecasting algorithm uses a two-level scheme composed by $(i)$ a time-series model to represent the daily aggregate flow values, and (ii) a set of different daily flow demand patterns according to the day type to cater for different consumption during the weekends and holidays periods. Every pattern consists of 24 hourly values for each daily pattern. This algorithm runs in parallel with the MPC algorithm. The daily series of hourly-flow predictions are computed as a product of the daily aggregate flow value and the appropriate hourly demand pattern. On the other hand, irrigation demand is typically planned in advance with farmers. Preestablished flows for irrigation are established in the irrigation areas for determined periods of the year.

\subsubsection{MPC problem formulation for water supply systems}

Water supply and distribution infrastructures are quite complex multivariate systems. In order to improve their performance, predictive control [8, 18] provides suitable techniques to compute optimal control strategies ahead in time for all the flow and pressure control elements of a water system. The optimal strategies are computed by optimizing a mathematical function describing the operational goals in a given time horizon and using a representative model of the network dynamics, as well as demand forecasts.

In many water systems, a supervision sampling time and control interval of the order of one hour is used, based on the scanning time of the telemetry system and on the dynamics of water distribution. Water consumption in urban areas is usually managed on a daily basis, because proper hourly 24-hour-ahead demand predictions may, in general, be available and common transport time delays between supply locations and the consumer sites allow operators to follow daily water request patterns. Therefore, this horizon is appropriate for evaluating the effects of different control strategies on the water network, with respect to operational goals. However, other horizons may also be appropriate for different utilities.

\section{Operational goals}

In most water systems, the regulated elements, namely pumps, gates and retention devices, are typically controlled locally, i.e., they are controlled by a remote station 
according to the measurements of sensors connected only to that station. However, a global real-time control system may require the use of an operational model of the system dynamics in order to compute, ahead in time, optimal control strategies for the actuators based on the current state of the system provided by supervisory control and data acquisition (SCADA) sensors, the current disturbance measurements and appropriate disturbance predictions. The computation procedure of an optimal global control law should take into account all the physical and operational constraints of the dynamical system, producing set-points though which given control objectives are achieved.

The immediate control goal of a water supply system is to meet the demands at consumer sites with appropriate flows, according to the requirements from the users. Predictive control techniques may be used to compute strategies which achieve this, while also optimizing the system performance in terms of different operational criteria. Regarding those criteria, some of them are discussed next.

- Cost reduction: The main economic costs associated with drinking water production are due to treatment processes, water acquisition or use costs and, most importantly, to electricity costs for pumping. Delivering this drinking water to appropriate pressure levels through the network involves important electricity costs in booster pumping as well as elevation from underground sources. In a specific case, this objective can be mathematically formulated as the minimization of

$$
J_{1}(k)=\left(\alpha_{1}+\alpha_{2}(k)\right) q_{u}(k),
$$

where $\alpha_{1}$ corresponds to a known vector related to the economic costs of the water depending on the selected water source, and $\alpha_{2}(k)$ is a vector of suitable dimensions associated to the economic cost of the flow through certain actuators (pumps only) and their control cost (pumping). Note the $k$-dependence of $\alpha_{2}$ since the pumping cost has different values according to the variable electric tariffs along a day.

- Operational safety: This criterion refers to maintaining appropriate water storage volumes in dams and tanks of the network for emergency handling. Therefore, this objective may be achieved by minimizing the following expression:

$$
J_{2}(k)= \begin{cases}\left(v(k)-v^{\text {safe }}\right)^{T}\left(v(k)-v^{\text {safe }}\right) & \text { if } \quad v(k) \leq v^{\text {safe }} \\ 0 & \text { otherwise }\end{cases}
$$

where $v^{\text {safe }}$ is a term which determines the safety volume to be considered for the control law computation.

- Demand management: This criterion refers to the fact that, although urban demands must be fully satisfied, irrigation demands allow some degree of slackness. Therefore, this objective may be achieved by minimizing the following expression:

$$
J_{3}(k)= \begin{cases}(q(k)-d(k))^{T}(q(k)-d(k)) & \text { if } q(k) \leq d(k) \\ 0 & \text { otherwise, }\end{cases}
$$


where $d(k)$ is the irrigation flow demanded for irrigation while $q(k)$ is the flow served.

- Minimum-flow management: This criterion refers to the need of enforcing that the generated flow strategies allow maintaining the appropriate water flows (or levels) in the river that allows to preserve the ecological flows and the navigability of the downstream portion of the river. This is achieved by means of the following objective expression:

$$
J_{4}(k)= \begin{cases}\left(q(k)-q^{\text {safe }}\right)^{T}\left(q(k)-q^{\text {safe }}\right) & \text { if } q(k) \leq q^{\text {safe }} \\ 0 & \text { otherwise }\end{cases}
$$

where $q^{\text {safe }}$ is a term which determines the safety flow to be considered for the control law computation.

- Control action smoothness: The operation of actuators usually requires smooth flow set-point variations for equipment conservation. To obtain such smoothing effect, control signal variation between consecutive time intervals (slew rate) is then penalized. The penalty term to be minimized is

$$
J_{5}(k)=\Delta q_{u}(k)^{T} \Delta q_{u}(k),
$$

where $\Delta q_{u}(k) \triangleq q_{u}(k)-q_{u}(k-1)$.

\section{Multi-objective performance function}

The multi-objective performance function $\mathcal{J}(k)$ that gathers the aforementioned control objectives may be written as

$$
\mathcal{J}(k)=\sum_{j=1}^{n_{J}} \gamma_{j} J_{j}(k)
$$

where a set of $n_{J}$ control objectives are considered and, in turn, a multi-objective open-loop optimization problem is stated. The prioritization of the control objectives is performed by using the order of the mathematical cost function associated to each objective, and also a set of appropriate weights $\gamma_{j}$. These weights are selected off-line by means of trial and error procedures, taking into account the priority of each objective within the cost function. More sophisticated tuning methodologies for tuning multi-objective control problems based on lexicographic minimizers [12], goal programming [4], or Pareto-front computations [22] may be also considered.

\section{Non-linear MPC strategy}

Collecting the control-oriented model and operational goals described in previous subsections, the MPC design follows the traditional procedures presented in 
[2, 8, 18], which involves solving an optimization problem over a prediction horizon $H_{p}$, where a cost function is minimized subject to a set of physical and operational constraints. Once the minimization is performed, a vector of control actions is obtained. Only the first component of that vector is considered and applied over the plant. The procedure is repeated for the next time instant taking into account the feedback measurements coming from the system, following the classic recedinghorizon strategy.

Given a general nonlinear dynamic model of the water system obtained applying the proposed control-oriented modeling methodology presented above

$$
x(k+1)=g(x(k), u(k)),
$$

being $x(k) \in \mathbb{R}^{n}$ the mapping of states and $u(k) \in \mathbb{R}^{m}$ the control signals, where $g: \mathbb{R}^{n} \times \mathbb{R}^{m} \rightarrow \mathbb{R}^{n}$ is an arbitrary system state function and $k \in \mathbb{Z}_{+}$, the MPC is based on the solution of the open-loop optimization problem

$$
\min _{\{u(k)\}_{i=0}^{H_{p-1}}} \sum_{i=0}^{H_{p}-1} \mathcal{J}(u(k+i \mid k), x(k+i \mid k)),
$$

subject to

$$
\begin{aligned}
& H u(k+i) \leq b, \\
& H_{e q} u(k+i)=b_{e q},
\end{aligned}
$$

$\forall i \in\left[0, H_{p}-1\right]$, where $\mathcal{J}(\cdot)$ is the cost function, $H_{p}$ denotes the prediction horizon, and $H, H_{e q}, b, b_{e q}$ are matrices with suitable dimensions. In sequence (2.17a), $x(k+$ $i \mid k$ ) denotes the prediction of the state at time $k+i$ performed at $k$, starting from $x(0 \mid k)=x(k)$. In particular, constraints $2.17 \mathrm{c}$ are related to elements with static dynamics, where an equality condition must hold. The optimal solution of (2.17) is given by the sequence

$$
u(0 \mid k)^{*}, u(1 \mid k)^{*}, \ldots, u\left(H_{p}-1 \mid k\right)^{*},
$$

and then the receding horizon philosophy sets

$$
u_{\mathrm{MPC}}(x(k)) \triangleq u(0 \mid k)^{*}
$$

disregarding the computed inputs from $k=1$ to $k=H_{p}-1$, with the whole process repeated at the next time instant $k \in \mathbb{Z}_{+}$. Expression 2.18) is known in the MPC literature as the MPC law.

In order to manage the uncertainty of the system disturbances over the prediction horizon, the stochastic paradigm can be used, which leads to include explicit models of uncertainty/disturbances in the design of control laws and by transforming hard constraints into probabilistic constraints. The stochastic approach is a classic one in the field of optimization, but due to the advances in technology which im- 
prove computation capacity and the flexibility of the MPC framework to incorporate models and constraints within an optimal control problem, a renewed attention has been given to the stochastic programming [20], as a powerful tool for robust control design, leading to stochastic MPC approaches.

\subsubsection{Case study: Guadiana river}

\section{Description}

As discussed in the introduction, the Guadiana has its source in Spain and flows into the Atlantic Ocean on the Portugal coast. It runs through the central south part of Spain from east to west most of its length to turn to the south direction when it is passing through Portugal. The hydrologic basin of Guadiana covers up to $67733 \mathrm{~km}^{2}$, from which $55512 \mathrm{~m}^{2}$ belongs to Spain $(81.9 \%)$. The annual average sources to the basin is about $3884 \mathrm{hm}^{3}$. The global basin has 87 reservoirs with higher capacity of $1 \mathrm{hm}^{3}$ and a total storage capacity of more than $9000 \mathrm{~m}^{3}$.

In the Guadiana catchment, the climatic conditions are characterized, in a general sense, by high temperature in summer and moderate in winter, and by a very irregular pluviometry, which is typical of the Mediterranean areas. This climatology configures a catchment that presents scarce and irregular rainfall, and sudden strong precipitation and frequent dry years. Agricultural and farming demand represents about the $90 \%$ of the water use while the remaining demand is domestic or industrial.

Currently, the monitoring and control of the Guadiana catchment is by the Automatic Hydrological Information System of the Guadiana Water Authority that, by means of an SCADA system (see Figure 2.6 for a sample of a SCADA screen), allows to know the current state of the water resources and river flows and operate dams and control elements. Currently, there are 144 control points: 86 in rivers sections, 48 in dams and 10 in canals plus 150 meteorological stations.

This chapter considers just the central part of this basin, where the Guadiana River is highly regulated by 16 dams linked by canals and pipes. The total capacity of these reservoirs is about $7800 \mathrm{hm}^{3}$, which regulate the river flow and supply to all the demands and the important irrigation zones of the basin. A bilateral agreement (Convenio de Albufeira) between Spain and Portugal establishes a minimum flow at specific sections of the river during normal precipitation years. This regulation takes into account the restrictions of ecological flows for summer periods and the operational rules for the reservoirs in rainy episodes. All these restrictions will be part of the optimization problem. The conceptual model of the Guadiana central portion is shown in Figure 2.5. In this figure, blue lines define the stretches rivers and the open canals, while in black lines define the pressurized pipes. 


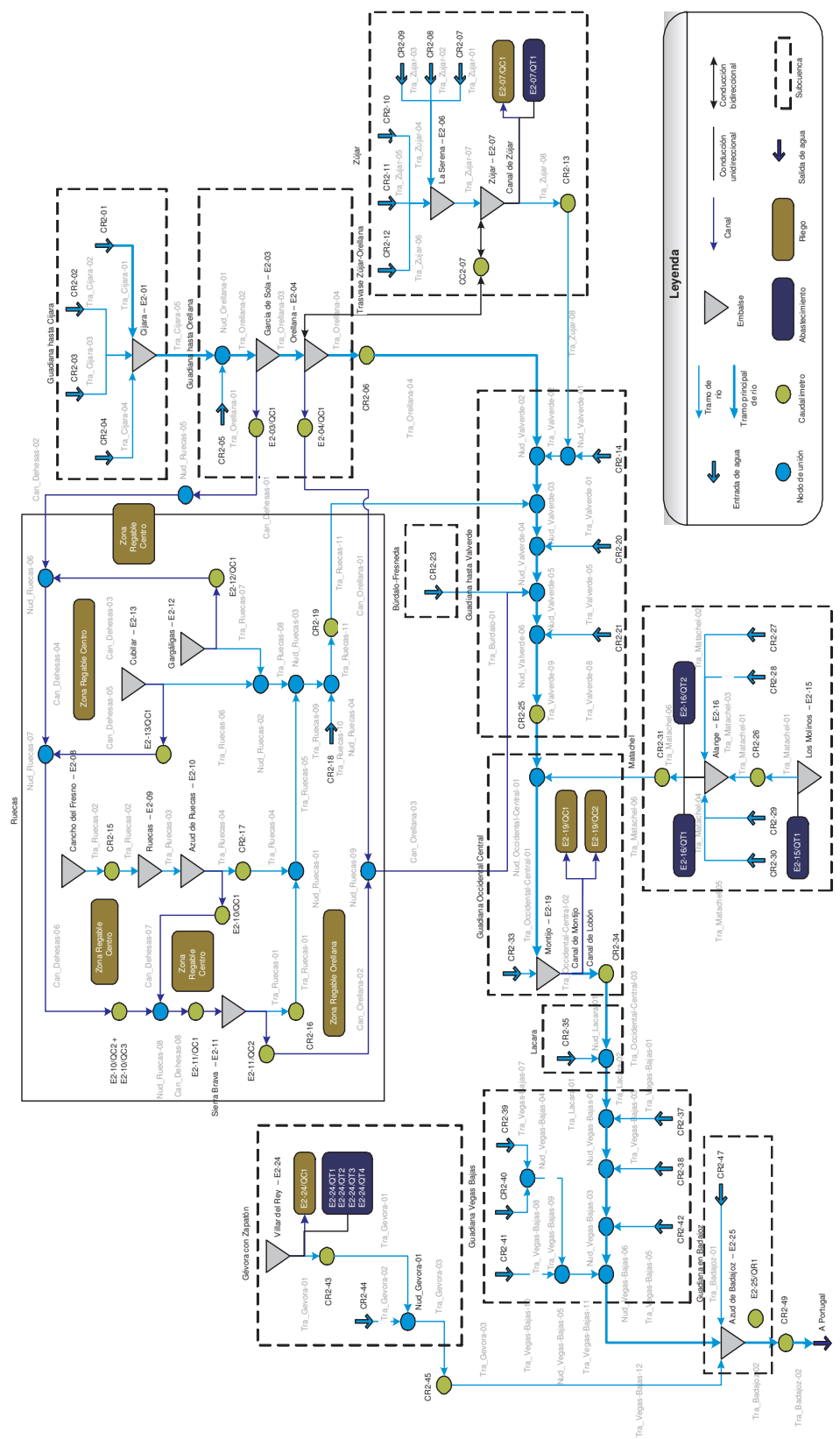

Fig. 2.5: Conceptual scheme of the Guadiana river portion used as case study. 


\section{Results}

In this section, control results are presented for representative selected elements. The control results have been obtained under the following considerations:

- The selected scenario lasts 16 months, with a sampling time of one day.

- The operational control strategy aim to guarantee the preservation of a minimum volume of water at the dams/reservoirs and the smooth behavior of the actuators (gates) while satisfying the consumer demands and the Convenio of Albufeira.

- The maximum available flow to deliver through all outlets of the reservoirs is limited by the total volume of water according to the corresponding discharge curve.

The control objectives of the multi-objective optimization associated to the MPC problem are defined in Section 2.2.2 The prediction horizon used for the MPC controller is one month (30 days). Optimization problems have been solved using the CONOPT3 solver in GAMS. The computer used to run the simulations is a PC Intel ${ }^{\circledR}$ Core ${ }^{\mathrm{TM}}$ running both cores at $2.8 \mathrm{GHz}$ with $4 \mathrm{~GB}$ of RAM. Computation time of each MPC iteration is less that one minute.

The MPC controller is implemented by means of the tool presented in Figure 2.7. This is general-purpose decision-support tool that has been developed to allow the user to implement optimal/predictive control techniques in large-scale water systems. An important feature of this tool compared to other existing tools is the application of a unified approach to the complete water system including supply, production, transport and distribution and, therefore pressurized and open-canal dynamics, simultaneously. The modeling and predictive control problem solution algorithm in this tool is designed for real-time decision support, in connection with the SCADA system using the OPC protocol. The hydraulic modeling relies on the control-oriented methodology described in Section 2.2.1. whose parameters are estimated on-line using recursive parameter estimation and real data from network sensors. Demand forecasting models, based on time-series analysis, are also dynamically updated. The real-time calibration using recursive parameter estimation methods contributes to deal with hydraulic uncertainty. This modeling choice, as well as the optimization method selection allow to deal with quite large-scale systems. Another distinguishing feature of the tool is its capability to accommodate complex operational goals.

Some results illustrating the performance of the proposed approach and developed tool in the Guadiana are presented in the following. Figure 2.8 shows the urban demand E2-07 supplied from the river. Figure 2.9 shows the resulting flow through gate V2-07 of a dam upstream of this demand, which is manipulated to satisfy it while fulfilling the rest of objectives such as the minimizing the river flow and maintaining the dam volume up to the safety volume. This can be seen in Figure 2.10 for the volume evolution of dam E2-07, where the control strategy guarantees that the dam volume surpasses the safety volume most of the time, being able to vary freely but without getting empty. On the other hand, Figure 2.10 shows that 


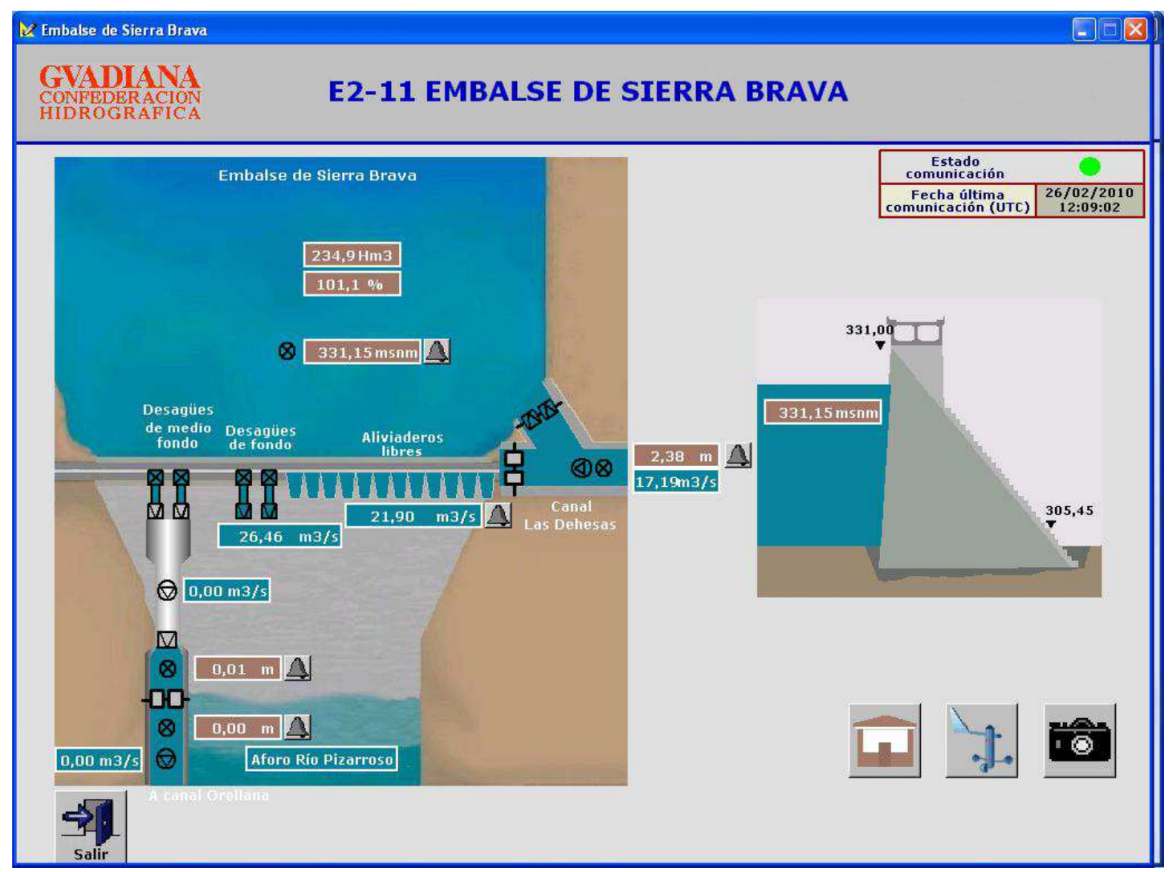

Fig. 2.6: Screen of the SCADA system.

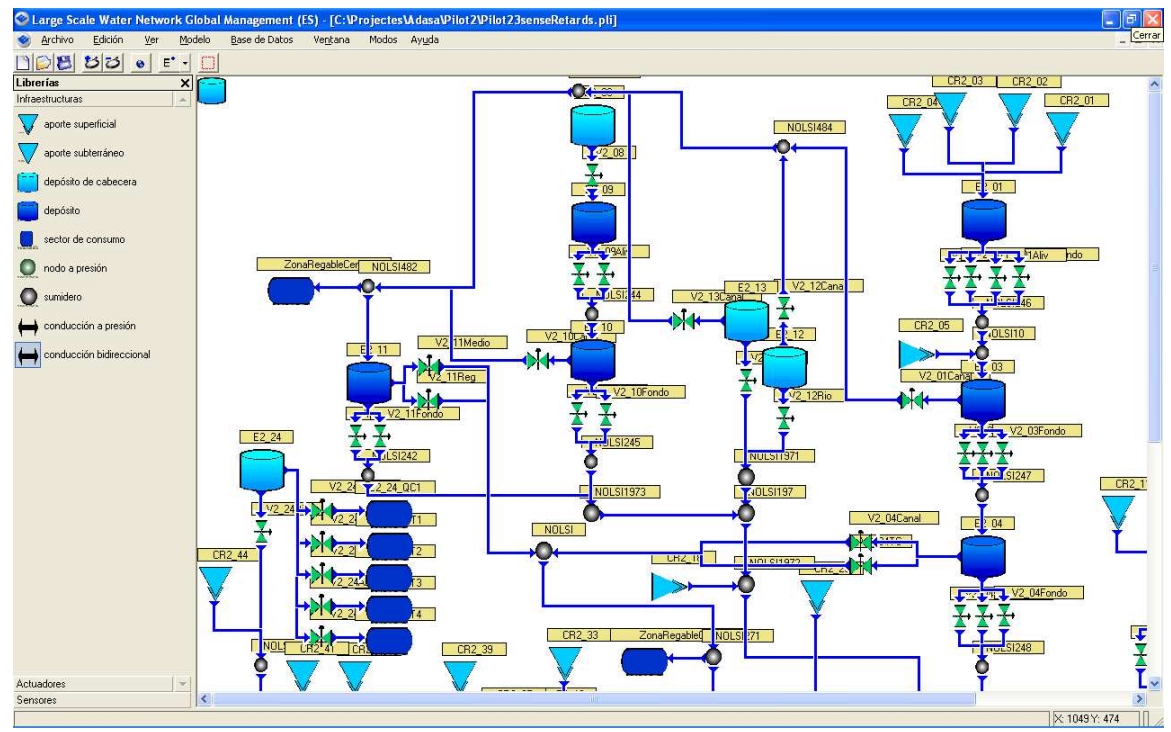

Fig. 2.7: Screen of the MPC design software tool. 


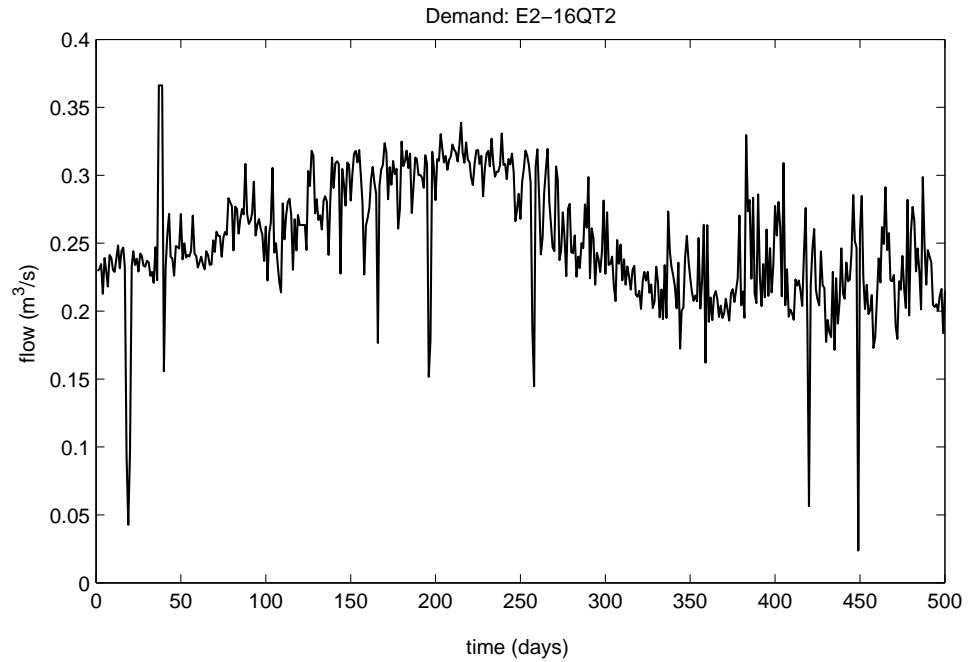

Fig. 2.8: Water demand E2-07.

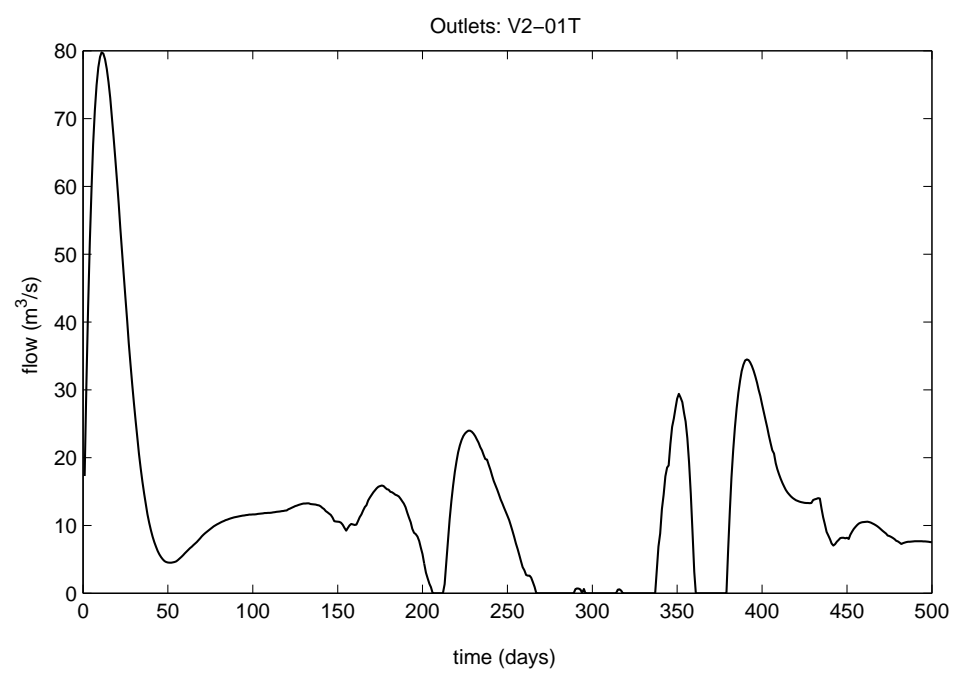

Fig. 2.9: Flow through gate V2-07.

the minimum flow $\left(2 \mathrm{~m}^{3} / \mathrm{s}\right)$ established by the Convenio of Albufeira to preserve sustainability and downstream navigability of the river is satisfied. 


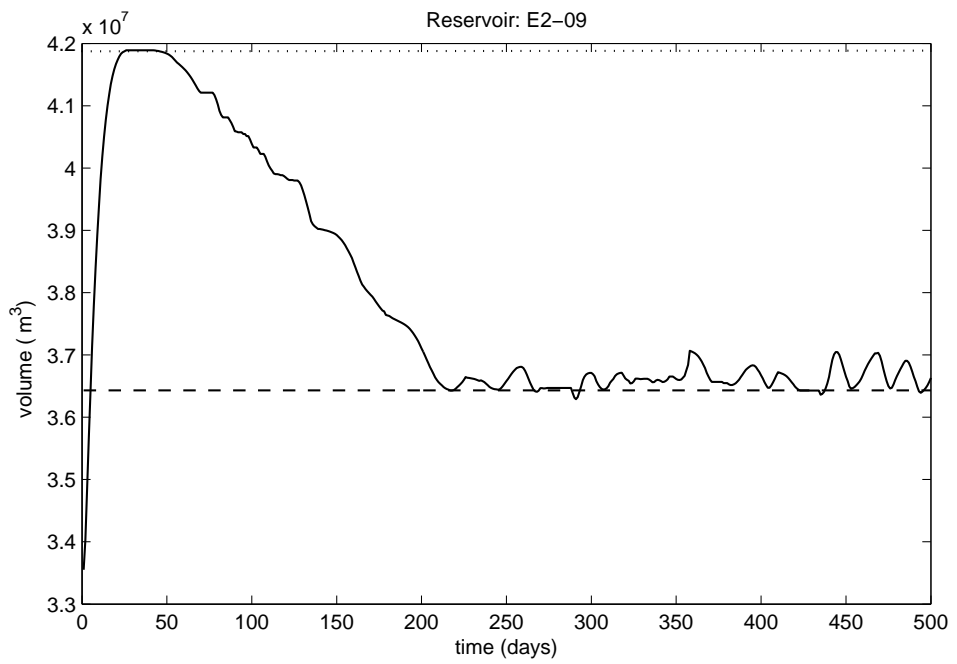

Fig. 2.10: Volume evolution of dam E2-07.
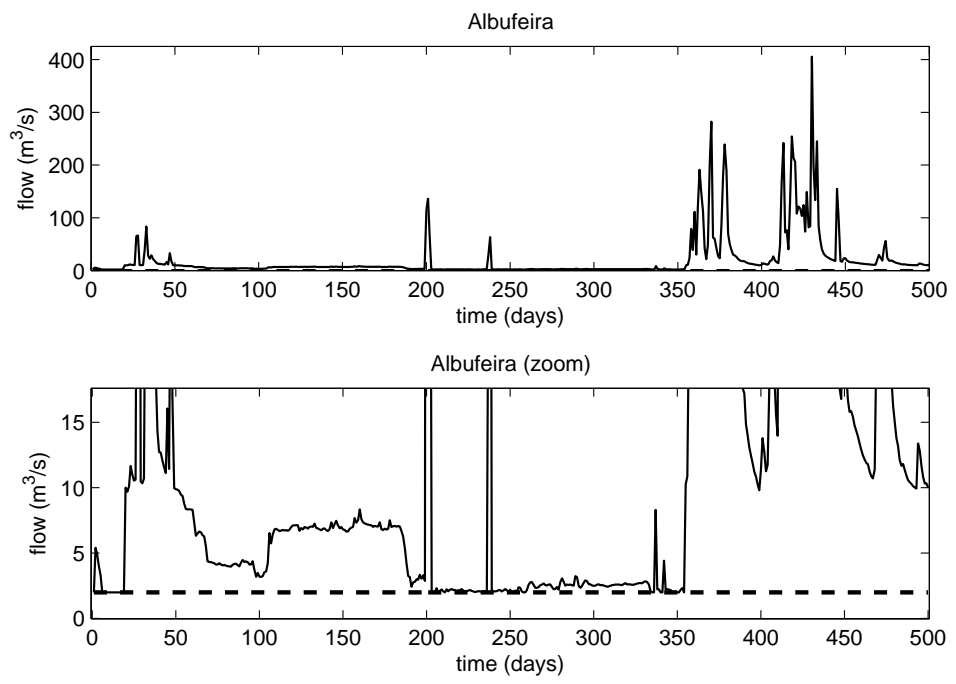

Fig. 2.11: Albufeira flow. 


\subsection{Linking transport of and transport over water}

As discussed along this chapter, an important characteristic of the Guadiana catchment are the strong seasonal variations as consequence of the inter-annual regularity of drought and rainy periods. This distinctive condition of the river implies that its flow needs to be regulated by means of dams in order to adapt it to the needs of the ecological and navigability flows ${ }^{1}$, hydroelectric generation and water supply demands. In drought periods, a great part of the Guadiana river tributaries have no water flow and show significant levels of eutrophication ${ }^{2}$. Until recently, the bilateral relationships between Spain and Portugal in water aspects were based on sharing water for hydroelectric generation. However, since November 1998, the Convenio de Albufeira agreement establishes a minimum flow at specific sections of the Guadiana river during normal precipitation years. This implies that integrated water resource management in all the Guadiana catchment is needed to manage in a efficient way the stored water in dams allowing to supply the required flows and keep the minimum flows at the specific sections of the Guadiana river. Nowadays, all this management is carried out using empirical rules and "historic" strategies, which come from years of operational experience and empirical results. While these may generally be adequate, the best operational policies may be quite complex to be determined for large-scale interconnected systems as the Guadiana catchment. Thus, decision-support systems for operational control as the one proposed in this chapter, based on mathematical models of network operation and optimal control techniques, provide useful guidance for efficient management of water networks at different levels (namely, as for the integrated water resources/watershed planning and management with medium or long-term horizons) and with contradictory goals (water supply and ecological/navigability flow preservation) specially in drought periods of scarce water resources.

\subsection{Conclusions}

In this chapter, a methodology based on MPC for the optimal management of combined water supply systems with navigability/sustainability features in river systems has been proposed. First, a control-oriented modeling approach for this type of systems has been introduced. Then, MPC is introduced as a means of generating flow control strategies from the sources to the consumer to meet future demands with appropriate flows, optimizing operational goals such as network safety volumes in dams and control input smoothness in actuators (valves, gates and pumps). At the same time, the resultant flow-control strategies allow maintaining the appropriate

\footnotetext{
${ }^{1}$ For the most part, the Guadiana is navigable until Mértola, a distance of $68 \mathrm{~km}$ away from the sea.

${ }^{2}$ Eutrophication or hypertrophication is defined as the ecosystem response to the addition of artificial or natural substances, mainly phosphates, through detergents, fertilizers, or sewage, to an aquatic system [19].
} 
levels in the river, allowing in turn to preserve the ecological flows and the navigability of the downstream portion of the river. The case study of Guadiana river has been used to illustrate the proposed management methodology. A decision support tool that implements the proposed methodology is described and some illustrative results in a real scenario are presented.

Acknowledgements This research has been partially funded by CDTI (MCyT) project HIDROPTIM IDI-20100722, the Spanish project ECOCIS (DPI-2013-48243-C2-1-R), the DGR of Generalitat de Catalunya (SAC group Ref. 2014 SGR 374), and the Maritime Project "ShipDrive: A Novel Methodology for Integrated Modeling, Control, and Optimization of Hybrid Ship Systems" (project 13276) of the Dutch Technology Foundation STW. The authors thank Confederación Hidrográfica del Guadiana for providing the case study as well as for sharing their hydrological management expertise.

\section{References}

[1] M. Brdys and B. Ulanicki. Operational Control of Water Systems: Structures, algorithms and applications. Prentice Hall International, UK, 1994.

[2] E.F. Camacho and C. Bordons. Model Predictive Control. Springer-Verlag, London, second edition, 2004.

[3] G. Cembrano, J. Quevedo, V. Puig, R. Pérez, J. Figueras, J. M. Verdejo, I. Escaler, G. Ramón, G. Barnet, P. Rodríguez, and M. Casas. Plio: A generic tool for real-time operational predictive optimal control of water networks. Water Science and Technology, 64(2):448-59, 2011.

[4] A. Chen and X. Xu. Goal programming approach to solving network design problem with multiple objectives and demand uncertainty. Expert Systems with Applications, 39(4):4160 - 4170, 2012.

[5] D. Kang. Real-time optimal control of water distribution systems. Procedia Engineering, 70(0):917 - 923, 2014.

[6] T. Koppel and A. Vassiljev. Estimation of real-time water fluxes in water distribution system on the basis of pressure measurements. Advances in Engineering Software, 66(0):19 - 23, 2013.

[7] X. Litrico and V. Fromion. Modeling and Control of Hydrosystems. Springer, 2009.

[8] J.M. Maciejowski. Predictive Control with Constraints. Prentice Hall, Great Britain, 2002.

[9] M. Marinaki and M. Papageorgiou. Optimal Real-time Control of Sewer Networks. Springer, Secaucus, NJ (USA), 2005.

[10] L.W. Mays. Urban Stormwater Management Tools. McGrawHill Professional Publishing, USA, 2004.

[11] C. Ocampo-Martinez, D. Barcelli, V. Puig, and A. Bemporad. Hierarchical and decentralised model predictive control of drinking water networks: Application to the Barcelona case study. IET Control Theory \& Applications, 6(1):62 - 71, 2012. 
[12] C. Ocampo-Martinez, A. Ingimundarson, V. Puig, and J. Quevedo. Objective prioritization using lexicographic minimizers for MPC of sewer networks. IEEE Transactions on Control Systems Technology, 16(1):113-121, 2008.

[13] C. Ocampo-Martinez, V. Puig, G. Cembrano, R. Creus, and M. Minoves. Improving water management efficiency by using optimization-based control strategies: the Barcelona case study. Water Science \& Technology: Water supply, 9(5):565-575, 2009.

[14] C. Ocampo-Martinez, V. Puig, G. Cembrano, and J. Quevedo. Application of predictive control strategies to the management of complex networks in the urban water cycle [applications of control]. IEEE Control Systems Magazine, 33(1):15-41, 2013.

[15] C. Ocampo-Martinez, V. Puig, J.M. Grosso, and S. Montes de Oca. DMPC made easy, chapter Multi-layer Decentralized Model Predictive Control of Large-Scale Networked Systems. Springer, 2013.

[16] P.J. Van Overloop. Model Predictive Control on Open Water Systems. Delft University Press, Delft, The Netherlands, 2006.

[17] J. Quevedo, V. Puig, G. Cembrano, and J. Blanch. Validation and reconstruction of flow meter data in the Barcelona water distribution network. Control Engineering Practice, 11(6):640-651, June 2010.

[18] J.B. Rawlings and D.Q. Mayne. Model Predictive Control: Theory and Design. Nob Hill Publishing, Madison, WI (USA), 2009.

[19] D. Schindler and J. Vallentyne. Algal Bowl: Overfertilization of the Worlds Freshwaters and Estuaries. University of Alberta Press, 2004.

[20] A. Shapiro, D. Dentcheva, and A. Ruszczynski. Lectures on stochastic programming: modeling and theory. Society for Industrial and Applied Mathematics and Mathematical Programming Society, 2009.

[21] R. Sitzenfrei and J. von Leon. Long-time simulation of water distribution systems for the design of small hydropower systems. Renewable Energy, 72(0): $182-187,2014$.

[22] R. Toro, C. Ocampo-Martinez, F. Logist, J. Van Impe, and V. Puig. Tuning of predictive controllers for drinking water networked systems. In Proceedings of the IFAC World Congress, Milano (Italy), 2011.

[23] Y. Wang, C. Ocampo-Martinez, V. Puig, and J. Quevedo. Gaussian-processbased demand forecasting for predictive control of drinking water networks. In Proceedings of the 9th International Conference on Critical Information Infrastructures Security, Limassol (Cyprus), October 13-15 2014.

[24] Y. Xu and X.Y. Zhang. Research on pressure optimization effect of high level water tank by drinking water network hydraulic model. Procedia Engineering, 31(0):958 - 966, 2012. 